\title{
The Tiebout Hypothesis Under Membership Property Rights
}

\author{
Goksel Asan and M. Remzi Sanver* ${ }^{\dagger}$
}

September 2007

\begin{abstract}
We consider the problem of producing an impure public good in various jurisdictions formed through the strategic decisions of agents. Our environment inherits two well-known problems:

(i) Under individual decisions, there is a tension between stability and efficiency;

(ii) Under coalitional decisions, stable jurisdiction structures may fail to exist.

The solution we propose is the use of membership property rights: When a move among jurisdictions is subject to the approval of the agents whom it affects, coalitionally stable jurisdiction structures coincide with those which are efficient.

Key Words: Tiebout hypothesis, coalition formation, membership property rights, impure public goods
\end{abstract}

JEL Classification Number: D71

\footnotetext{
*Department of Economics, İstanbul Bilgi University; corresponding author: gasan@bilgi.edu.tr

${ }^{\dagger}$ This paper has been presented at the Eighth International Meeting of thr Association for Public Economic Theory, 6-8 July 2007, Nashville and the 29'th Bosphorus Workshop on Economic Design, 25 August-1 September 2007, Bodrum. We thank the participants. Remzi Sanver acknowledges the support of the Turkish Academy of Science Distinguished Young Scientist Award Program (TUBA-GEBİP)
} 


\section{Introduction}

We consider a society confronted to the problem of producing a public good through the private contributions of its members. The public good is impure, i.e., exhibits negative crowding effect. As a result, a coalition of agents may have the incentive of splitting, so as to form a jurisdiction that produces its own local public good which cannot be consumed by outsiders. It is thus possible to observe a partition of the society- to which we refer as a jurisdiction structure- where each jurisdiction produces the public good locally. We address the relationship between the stability and efficiency of jurisdiction structures.

The problem we pose belongs to the theory of local public goods that goes back to Tiebout (1956) who suggests that agents reveal their preferences through their choice of jurisdictions, thus ensuring that "equilibrium" jurisdiction structures are Pareto optimal. This claim is stated in an informal setting, hence cannot be formally falsified. Nevertheless, the literature following Tiebout (1956) is quite rich in presenting cases against the efficiency of "equilibrium" jurisdiction structures. ${ }^{1}$

The definition of efficiency being rather standard, it is the meaning attributed to "equilibrium" that is critical in determining the relation between the two concepts. A standard equilibrium notion -which is usually called freemobility equilibrium- is the immunity requirement against individual deviations. This is quite a weak requirement which may end up in inefficient jurisdiction structures. $^{2}$ An extreme strengthening of free-mobility equilibria is the immunity requirement against coalitional deviations. This is what Greenberg and Weber (1986) call strong Tiebout equilibrium. ${ }^{3}$ Although strong Tiebout equilibria are typically efficient, the strength of the notion results in its possible failure to exist. ${ }^{4}$ As a solution to this trade-off, Conley and Konishi (2002) propose migration-proof Tiebout equilibrium as a solution concept whose strenght lies between free mobility and strong Tiebout equilibria. They show for sufficiently large economies with homogeneous agents that a migration-proof Tiebout equilibrium uniquely exists and it is asymptotically efficient.

The solution we propose has a different perspective: We explore the effects of introducing membership property rights so that a move between jurisdictions may require the consent of agents who are not directly involved in that move. ${ }^{5}$

\footnotetext{
${ }^{1}$ As a famous example in this direction, we have the critique of Bewley (1981) who shows that equilibrium district structures may fail to exist or be inefficient.

${ }^{2}$ Buchanan and Goetz (1972) and Flatters et al. (1974) are among the first to point to the possible inefficiency of free-mobility equilibria. The literature contains many explorations about this particular equilibrium concept, among which we have Richter (1982), Greenberg (1983), Konishi (1996) and Konishi et al. (1998).

${ }^{3}$ The idea of being immune to coalitional deviations has been previously used by McGuire (1974) and Wooders (1978).

${ }^{4}$ Guesnerie and Oddou (1981) and Greenberg and Weber $(1986,1993)$ discuss the existence conditions of strong Tiebout equilibria. The existence of coalitionally stable partitions in an abstract hedonic setting is addressed by Banerjee et al. (2001), Bogomolnaia and Jackson (2002).

${ }^{5}$ We owe this concept to Sertel (1992) whose formulation in an abstract setting has been worked on by Eren (1993) and further treated in detail by Sertel $(1998,2003)$. The concept has
} 
A membership property rights code assigns to each move, the list of agents whose approval is necessary for that move to occur. Thus, the stability of a jurisdiction structure depends on the used equilibrium concept as well as the membership property right code. This raises an interesting economic design problem which is the subject matter of this paper: Can the membership property right code be set so that the tension between stability and efficiency vanishes?

In Section 2, we introduce the model. Our environment allows any finite society with heterogeneous agents - hence being less restrictive than the one conceived by Conley and Konishi (2002). We assume that the public good level within a jurisdiction is determined by the voluntary contributions of the jurisdiction members. ${ }^{6}$ Each jurisdiction consumes its own public good - thus our model is also associated with the non-cooperative theory of coalition formation where coalitions are hedonic. ${ }^{7}$ Section 3 collects the results under free mobility equilibria. These are rather negative. As an unsurprising fact, when individuals can freely exit from and enter to jurisdictions, neither stable jurisdiction structures have to be efficient, nor efficient ones are necessarily stable. In fact, as a stronger observation, there are economies where stable and efficient jurisdiction structures form disjoint sets. This tension between stability and efficiency can be partially relaxed through the introduction of membership property rights. Under approved entry ${ }^{8}$ and approved exit ${ }^{9}$, every efficient jurisdiction structure is stable. Nevertheless, the existence of stable but inefficient jurisdiction structures prevails. In Section 4, we allow for coalitional moves. Unsurprisingly, coalitionally stable jurisdiction structures are always efficient but they may fail to exist. However, by an appropriate choice of the membership property right code, one can ensure the coalitional stability of efficient jurisdiction structures. More precisely, under approved entry and approved exit, coalitionally stable and efficient jurisdiction structures coincide - hence rehabilitating the Tiebout (1956) hypothesis. Section 5 makes some closing remarks.

been applied to more structured frameworks, such as the worker-partnership model proposed by Sertel (1982); the pure public good production problem analyzed by Asan and Sanver (2003) and the coalition formation analysis of Ozkal-Sanver (2005), Nizamogullari and Ozkal-Sanver (2007) in matching problems.

${ }^{6}$ Bergstrom et al. (1986) give a detailed analysis of the voluntary contributions solution (VCS) which is well-known to pave the way to inefficient allocations. Our choice of VCS as the prevailing allocation rule is rather arbitrary. It can be justified by assuming the nonexistence of institutions which implement efficient allocation rules. After all, our interest is not towards the efficiency of the considered public good allocation rule, but towards the efficiency of institutions leading to jurisdiction formations under a given allocation rule. Moreover, we conjecture that our results prevail under any allocation rule which satisfies the population monotonicity condition used by Sertel and Yildiz (1998).

${ }^{7}$ We owe this terminology to Drèze and Greenberg (1980) who call the dependence of an agent's payoff to only the members of his coalition the "hedonic aspect". A thorough analysis of hedonic coalition structures is made by Bogomolnaia and Jackson (2002).

8 i.e., the entrance to a jurisdiction requires the consent of all members of that jurisdiction.

9 i.e., the exit from a jurisdiction requires the consent of all members of that jurisdiction. 


\section{The Model}

\subsection{Basic notions}

We pick an integer $n \geq 2$ and consider a society $N=\{1, \ldots, n\}$, confronted to the problem of providing an (impure) public good by the private contributions of its agents. The economy consists of a single public and a single private good. While all agents consume the same amount of public good $y \in \Re_{+}$, we write $x_{i} \in \Re_{+}$for the amount of private good allocated to agent $i \in N$. Agents have preferences over the private good-public good bundles they consume, as well as the number of agents with whom they share the public good. So every $i \in N$ has a real-valued utility function $u_{i}\left(x_{i}, y, k\right)$ where $\left(x_{i}, y\right) \in \Re_{+} \times \Re_{+}$is an allocation and $k \in\{1, \ldots, n\}$ is the number of agents simultaneously consuming $y$. We write $U$ for the set of such utility functions which satisfy the following two conditions:

(i) Fixing any $k \in\{1, \ldots, n\}, u_{i}\left(x_{i}, y, k\right)$ is continuous, monotonically increasing and strictly quasi-concave with respect to $\left(x_{i}, y\right)$.

(ii) Fixing any $\left(x_{i}, y\right) \in \Re_{+} \times \Re_{+}, u_{i}\left(x_{i}, y, k\right)$ is non-increasing in $k$.

Every $i \in N$ is endowed by zero public good and a strictly positive amount of private good denoted by $\omega_{i}$. Thus each $i \in N$ is characterized by an ordered pair $\left(\omega_{i}, u_{i}\right) \in \Re_{++} \times U$. The family $e=\left\{\left(\omega_{i}, u_{i}\right)\right\}_{i \in N}$ of such ordered pairs is an economy and $E$ is the set of all economies where both the private and public good are normal.

\subsection{Production of the public good}

At any $e \in E$, the public good level equals the total amount of private good contributions. So denoting $t_{i}$ the private good contribution of $i \in N$, we have $y=\sum_{i \in N} t_{i}$.

We assume that a non-empty coalition $K \subseteq N$ of agents may come together to produce and consume their own public good, isolated from the rest of the society. We refer to such a coalition as a jurisdiction. Any jurisdiction $K$ produces its own public good by the voluntary contributions of its members, which results in a game $\Gamma_{K}=\left\{\left(\Theta_{i}, p_{i}\right)\right\}_{i \in K}$ where $\Theta_{i}=\left[0, \omega_{i}\right]$ is the strategy space of $i \in K$. The joint strategy space of $\Gamma_{K}$ is denoted as $\Theta=\prod_{i \in K} \Theta_{i}$ and any $i \in K$ has a preference over $\Theta$ represented by the real valued function $p_{i}: \Theta \rightarrow$ $\Re$, defined for any $t \in \Theta$ as $p_{i}(t)=u_{i}\left(\omega_{i}-t_{i}, \sum_{j \in N} t_{j}, \# K\right)$. Each $i \in K$ chooses some contribution $t_{i}^{*} \in \Theta_{i}$ such that $t_{i}^{*}=\operatorname{argmax}_{t_{i} \in \Theta_{i}} u_{i}\left(\omega_{i}-t_{i}, t_{i}+T_{-i}, \# K\right)$ where $T_{-i}=\sum_{j \in N \backslash\{i\}} t_{j}$ denotes the total contributions of the agents other than $i$. For any $K \subseteq N$, let $V(K)=\left(x_{1}^{*}, \ldots, x_{\# K}^{*}, y^{*}\right) \in \Re_{+}^{N} \times \Re_{+}$be the allocation induced by the Nash equilibrium vector $\left(t_{1}^{*}, \ldots, t_{\# K}^{*}\right) \in \Theta$ of the game $\Gamma_{K}$ such 
that $y^{*}=\sum_{j \in N} t_{j}^{*}$ and $x_{i}^{*}=\omega_{i}-t_{i}^{*} \forall i \in K$. We call $V(K)$ the voluntary contributions allocation of the jurisdiction $K .{ }^{10}$ We write $V_{i}(K)=\left(x_{i}^{*}, y^{*}, \# K\right)$ for the outcome enjoyed by $i \in K$, a result of the voluntary contributions allocation.

\subsection{Formation of jurisdictions: Individual moves}

We let $\Pi(K)$ stand the set of all possible partitions of $K \in 2^{N} \backslash\{\emptyset\} .{ }^{11}$ We simplify notation by writing $\Pi$ instead of $\Pi(N)$. Any $\pi \in \Pi$ is called a jurisdiction structure (of the society). At each $\pi \in \Pi$, agents belonging to the same jurisdiction $K \in \pi$ produce their own public good, consumed only by themselves. So each $i \in N$ has a preference over $\Pi$ represented by a real-valued utility function $v_{i}: \Pi \rightarrow \Re$ defined for each $\pi \in \Pi$ as $v_{i}(\pi)=u_{i}\left(V_{i}(\pi(i))\right)$ where $\pi(i) \in \pi$ stands for the jurisdiction to which agent $i$ belongs in the jurisdiction structure $\pi$.

For any $K, L \subseteq N$ and any $i \in N$, we introduce a function $F_{i, K, L}: \Pi \rightarrow \Pi$ which is defined for each $\pi \in \Pi$ as follows:

$$
F_{i, K, L}(\pi)=\left\{\begin{array}{cc}
(\pi \backslash\{K, L\}) \cup\{K \backslash\{i\}, L \cup\{i\}) & \text { if } K=\pi(i) \neq\{i\} \text { and } L \in \pi \\
(\pi \backslash\{K, L\}) \cup\{L \cup\{i\}) & \text { if } K=\pi(i)=\{i\} \text { and } L \in \pi \\
(\pi \backslash\{K\}) \cup\{K \backslash\{i\},\{i\}) & \text { if } K=\pi(i) \neq\{i\} \text { and } L=\varnothing \\
\pi & \text { otherwise }
\end{array}\right.
$$

So $F_{i, K, L}(\pi)$ is the jurisdiction structure obtained from $\pi$ by the move of agent $i$ from jurisdiction $K$ to jurisdiction $L$. Remark that defining such a move makes sense only if $i \in K$ and otherwise we let $F_{i, K, L}(\pi)=\pi$. On the other hand $L=\varnothing$ is allowed, as $i$ can move to an "empty jurisdiction". However, in case $K=\{i\}$ and $L=\varnothing$, we have $F_{i, K, L}(\pi)=\pi$.

But who has the right to accept or refuse such moves? Following Sertel (2003), for each possible individual move, we define a "code", which is the list of agents who must be consulted for this move to take place: Given any $K \in$ $2^{N} \backslash\{\emptyset\}$, any $i \in K$ and any $L \in 2^{N}$ with $K \cap L=\varnothing$, we write $C_{i, K, L} \subseteq N$ for the set of agents who must be consulted when agent $i$ wants to leave jurisdiction $K$ and enter the (possibly empty) jurisdiction $L$. The family $C=\left\{C_{i, K, L}\right\}$ is the membership property right code of the society. We explicitly define $C$ under four membership property right axioms: For each $K, L \subseteq N$ with $K \cap L=\varnothing$ and each $i \in K$, we have

- Free Entry - Free Exit (FE-FX) iff $C_{i, K, L}=\{i\}$

- Approved Entry - Free Exit (AE-FX) iff $C_{i, K, L}=\{i\} \cup L$

\footnotetext{
${ }^{10}$ Bergstrom et al. (1986) show that this will be unique in our assumed environment. From now on we let $\mathrm{E}$ be the set of economies (with normal private and public goods) where any $K \subseteq N$ leads to a voluntary contributions allocation $V(K)$ with $y^{*}>0$, i.e., there is at least one agent who contributes to the public budget.

${ }^{11} \mathrm{~A}$ partition of $K$ is a finite family $\left\{K_{t}\right\}$ of pairwise disjoint subsets of $K$ such that $\cup K_{t}=K$. While the usual definition requires each $K_{t}$ to be non-empty, we relax the definition by treating the partitions $\left\{K_{t}\right\}$ and $\left\{K_{t}\right\} \cup\{\emptyset\}$ equivalently.
} 
- Free Entry - Approved Exit (FE-AX) iff $C_{i, K, L}=K$

- Approved Entry - Approved Exit (AE-AX) iff $C_{i, K, L}=K \cup L$.

A jurisdiction structure $\pi \in \Pi$ is said to be individually stable under a membership property right code $C$ iff for any $i \in N$ and any $L \in(\pi \backslash\{\pi(i)\}) \cup$ $\{\varnothing\}$, we have $v_{i}(\pi) \geq v_{i}\left(F_{i, \pi(i), L}(\pi)\right)$ or $v_{j}(\pi)>v_{j}\left(F_{i, \pi(i), L}(\pi)\right)$ for some $j \in$ $C_{i, \pi(i), L}$. Under FE-FX, the existence of individually stable partitions is not guaranteed over $E$. To see this, for any positive real number $r$, let $(r)^{+}$(resp., $\left.(r)^{-}\right)$stand for some real number higher (resp. lower) than but "sufficiently close to" $r$. Now take $N=\{1,2\}$ and let $e \in E$ be such that $\omega_{i}=1 \forall i \in N$, $u_{1}\left(x_{1}, y, k\right)=x_{1}, y$ for $k \in\{1,2\}$ while $u_{2}\left(x_{2}, y, 1\right)=x_{2} . y$ and $u_{2}\left(x_{2}, y, 2\right)=$ $\frac{x_{2} \cdot y}{\left(\frac{16}{9}\right)^{+}}$. Check that $v_{1}(\{1\},\{2\})=\frac{1}{4}$ and $v_{1}(\{N\})=\frac{4}{9}$ while $v_{2}\left(\{\{1\},\{2\})=\frac{1}{4}\right.$ and $v_{1}(\{N\})=\left(\frac{1}{4}\right)^{-}$, showing that under FE-FX none of the logically possible partitions is stable. ${ }^{12}$

Remark 2.1 Extending the list of agents who must be consulted for the move of some $i \in N$ from a jurisdiction $K$ to a jurisdiction $L$ has an enlarging effect on the set of stable jurisdiction structures. In other words, given any two membership property right codes $C$ and $C^{\prime}$ where $C_{i, K, L} \subseteq C_{i, K, L}^{\prime}$ for all $K, L \subseteq N$ with $K \cap L=\varnothing$ and for all $i \in K$, the stability of $\pi \in \Pi$ under $C$ implies the stability of $\pi$ under $C^{\prime}$.

\subsection{Formation of jurisdictions: Coalitional moves}

Given any $\pi \in \Pi$, any non-empty $S \subseteq N$ and any $\rho \in \Pi(S)$, the $\rho$-move of $S$ is the jurisdiction structure $F_{S, \rho}(\pi)=\left\{T \backslash S \in 2^{N}: T \in \pi\right\} \cup \rho{ }^{13}$ So all members of $S$ leave their jurisdiction at $\pi$, they partition according to $\rho$ and each $S^{\prime} \in \rho$ moves to an "empty jurisdiction". Remark that coalitional moves generalize individual moves. It is clear that the (individual) move of $i \in N$ from $\pi(i)$ to the empty jurisdiction corresponds to the $\rho$-move of $\{i\}$ where trivially $\rho=\{\{i\}\}$. In a similar vein, the move of $i$ from $\pi(i)$ to $L \in \pi$ is equivalent to the $\rho$-move of $L \cup\{i\}$ where $\rho=\{L \cup\{i\}\}$.

We say that $S$ blocks $\pi \in \Pi$ iff there exists $\rho \in \Pi(S)$ such that $v_{i}\left(F_{S, \rho}(\pi)\right) \geq$ $v_{i}(\pi) \forall i \in S$ while $v_{i}\left(F_{S, \rho}(\pi)\right)>v_{i}(\pi)$ for some $i \in S$. Any $\rho$-move of a coalition $S$ is subject to the approval of those agents specified by the membership property right code $C=\left\{C_{i, K, L}\right\}$ where again $C_{i, K, L} \subseteq N$ is the set of agents who must be consulted when agent $i \in K$ wants to leave jurisdiction $K$ to enter

\footnotetext{
${ }^{12}$ Individual stability of $\pi \in \Pi$ under FE-FX is equivalent to the free mobility equilibrium as defined in Conley and Konishi (2002). Bogomolnaia and Jackson (2002) identify, in a general hedonic setting, conditions that ensure the existence of individually stable partitions. Note that individual stability under FE-FX (resp, AE-FX, AE-AX) is called Nash stability (resp., individual stability, contractual individual stability) by Bogomolnaia and Jackson (2002).

${ }^{13} T \backslash S$ is empty when $T \subseteq S$. This violates the usual definition of a partition. However, as noted in Footnote 11, we allow partitions to include the empty-set.
} 
jurisdiction $L$. Nevertheless, under our definition of a coalitional move, $L$ is always empty. So for coalitional moves, it is $\left\{C_{i, K, \emptyset}\right\}$ which matters.

A jurisdiction structure $\pi \in \Pi$ is said to be coalitionally stable under a membership property right code $C$ iff

(i) For any $S \in 2^{N} \backslash\{\emptyset\}$ which blocks $\pi$ by some $\rho$-move, there exists $i \in S$ such that $v_{j}(\pi)>v_{j}\left(F_{S, \rho}(\pi)\right)$ for some $j \in(\pi(i) \backslash S) \cap C_{i, \pi(i), \emptyset}$

and

(ii) $\pi \in \Pi$ is individually stable under $C .{ }^{14}$

\section{Results: Individual Stability}

We start by recalling the case where the public good exhibits no crowding effect. Let $E^{p} \subset E$ be the set of economies where the public good is pure, i.e., for every $i \in N$ and every $\left(x_{i}, y\right) \in \Re_{+} \times \Re_{+}$, we have $u_{i}\left(x_{i}, y, k\right)=u_{i}\left(x_{i}, y, k^{\prime}\right)$ $\forall k, k^{\prime} \in\{1, \ldots, n\}$. The following result is quoted from Asan and Sanver (2003):

Theorem 3.1 Let the membership property right code be determined by FE-FX. At every $e \in E^{p}$, there exists a unique individually stable jurisdiction structure as well as a unique efficient ${ }^{15}$ jurisdiction structure which is $\{N\}$.

So under the absence of crowding effects, the jurisdiction structure $\{N\}-$ to which we refer as the grand jurisdiction- is efficient and individually stable, independent of the economy. Hence, we have a counterpart of the first theorem of welfare economics (every stable jurisdiction structure is efficient) as well as of the second one (every efficient jurisdiction structure is stable).

Remark 3.1 Remark 2.1 advices some caution about the fate of Theorem 3.1 when the membership property right code is tightened. As Asan and Sanver (2003) show, Theorem 3.1 remains intact when FE is replaced by AE. On the other hand, switching from $F X$ to AX leads to new individually stable partitions, which ends up in individually stable but inefficient jurisdiction structures - hence leading to a failure of the first welfare theorem.

The stability-efficiency harmony announced by Theorem 3.1 is due to the absence of crowding effects. Once we leave the world of pure public goods, there is no reason to expect the grand jurisdiction to be the only efficient one, or even to be efficient. ${ }^{16}$ In fact, when the public good is impure, no jurisdiction

\footnotetext{
${ }^{14}$ The fact that coalitional moves generalize individual moves does not imply that coalitional stability generalizes individual stability. For, conceiving the move of $i$ from $\pi(i)$ to $L \in \pi$ as the $\rho$-move of $L \cup\{i\}$ imposes an implicit approved entry requirement to the individual move of $i$, as no member of $L \cup\{i\}$ must be worsed-off by the $\rho$-move of $L \cup\{i\}$. Note that coalitional stability of $\pi \in \Pi$ under FE-FX is stronger than the Conley and Konishi (2002) definition of strong Tiebout equilibrium.

${ }^{15}$ We say that $\pi \in \Pi$ is efficient iff there exists no $\pi^{\prime} \in \Pi$ such that $v_{i}\left(\pi^{\prime}\right) \geq v_{i}(\pi) \forall i \in N$ and $v_{i}\left(\pi^{\prime}\right)>v_{i}(\pi)$ for some $i \in N$. Due to the finiteness of the society (hence the finiteness of the logically possible partitions), every $e \in E$ admits an efficient jurisdiction structure.

${ }^{16}$ The intuition behind this claim -which we will justify soon- is related to the seminal work of Buchanan (1965) which determines the optimal size of jurisdictions.
} 
structure can a priori be classified as individually stable or efficient - a result which we state below:

Theorem 3.2 Take any jurisdiction structure $\pi \in \Pi$. There exists e $\in E$ where

(i) $\pi$ is efficient

(ii) $\pi$ is not efficient

(iii) $\pi$ is individually stable under FE-FX

(iv) $\pi$ is not individually stable under FE-FX

Proof. Take any $\pi \in \Pi$. To see $(i)$, consider first the case where $\pi$ is the grand jurisdiction. We know, by Theorem 3.1, that $\pi$ is efficient when $e \in E^{p}$. Now consider the case where $\pi$ is not the grand jurisdiction. Take any $e \in E$ such that $\omega_{i}=\omega_{j} \forall i, j \in N$ while for each $i \in N$ we have $u_{i}\left(x_{i}, y, k\right)=\left\{\begin{array}{l}x_{i} \cdot y \text { when } k \leq \# \pi(i) \\ \frac{x_{i} \cdot y}{M} \text { when } k>\# \pi(i)\end{array}\right.$ where $M$ is a positive real number. Picking $M$ sufficiently large, ensures the efficiency of $\pi$. To see (ii), consider first the case where $\pi$ is the grand jurisdiction. Take any $e \in E$ such that $\omega_{i}=\omega_{j}$ $\forall i, j \in N$ while for each $i \in N$ we have $u_{i}\left(x_{i}, y, k\right)=\left\{\begin{array}{l}x_{i} \cdot y \text { when } k=1 \\ \frac{x_{i} \cdot y}{M} \text { when } k>1\end{array}\right.$ where $M$ is a positive real number. Picking $M$ sufficiently large ensures that $\pi$ is not efficient. Now consider the case where $\pi$ is not the grand jurisdiction. We know, by Theorem 3.1, that $\pi$ is not efficient when $e \in E^{p}$. To see (iii), assume FE-FX. Consider first the case where $\pi$ is the grand jurisdiction which, by Theorem 3.1, is stable at any $e \in E^{p}$. Now consider the case where $\pi$ is not the grand jurisdiction. Take any $e \in E$ such that $\omega_{i}=\omega_{j} \forall i, j \in N$ while for each $i \in N$ we have $u_{i}\left(x_{i}, y, k\right)=\left\{\begin{array}{l}x_{i} \cdot y \text { when } k \leq \# \pi(i) \\ \frac{x_{i} \cdot y}{M} \text { when } k>\# \pi(i)\end{array}\right.$ where $M$ is a positive real number. Picking $M$ sufficiently large ensures the stability of $\pi$. To see (iv), assume FE-FX. Consider first the case where $\pi=\{\{i\}: i \in N\}$ which, by Theorem 3.1, fails to be stable at any $e \in E^{p}$. Consider now the case where $\pi$ differs from $\{\{i\}: i \in N\}$. Take any $e \in E$ such that $\omega_{i}=\omega_{j} \forall i, j \in N$ while for each $i \in N$ we have $u_{i}\left(x_{i}, y, k\right)=\left\{\begin{array}{l}x_{i} . y \text { when } k=1 \\ \frac{x_{i} \cdot y}{M} \text { when } k>1\end{array}\right.$ where $M$ is a positive real number. Picking $M$ sufficiently large ensures that $\pi$ is not stable.

Remark 3.2 Theorem 3.2 remains intact when we switch from FE to AE or from FX to $A X$. The efficiency of a jurisdiction structure does not depend on the membership property right code - hence parts (i) and (ii) prevail. The fact announced by Remark 2.1 ensures this for part (iii). To see this for part (iv), it suffices to check that the non-stability exhibited by the examples used in the proof does not depend on the membership property right code.

Our next result announces the existence of economies where stable and efficient jurisdiction structures form disjoint sets - hence the failure of both welfare theorems:

Theorem 3.3 There exists $e \in E$ where under FE-FX, the (non-empty) sets of stable and efficient jurisdiction structures are disjoint. 
Proof. Consider $e \in E$ where $N=\{1,2,3,4\}$ and $\omega_{i}=1 \forall i \in N$. Letting $c_{1}=\left(\frac{81}{64}\right)^{+}$and $c_{2}=2$, preferences of agents are

$$
\begin{aligned}
& u_{i}\left(x_{i}, y, k\right)=\left\{\begin{array}{c}
x_{i} \cdot y \text { when } k \leq 2 \\
\frac{x_{i} \cdot y}{c_{1}} \text { when } k=3 \\
\frac{x_{i} \cdot y}{c_{2}} \text { when } k=4
\end{array} \text { wh } i \in\{1,2\}\right. \text { and } \\
& u_{i}\left(x_{i}, y, k\right)=\left\{\begin{array}{c}
x_{i} \cdot y \text { when } k \leq 3 \\
\frac{x_{i} \cdot y}{c_{2}} \text { when } k=4
\end{array} \text { for } i \in\{3,4\} .\right. \text { One can check that this }
\end{aligned}
$$
implies for each $\pi \in \Pi$ and each $i \in\{1,2\}$,

$$
\begin{aligned}
& v_{i}(\pi)=\left\{\begin{array}{c}
\frac{1}{4} \text { when } \# \pi(i)=1 \\
\frac{4}{9} \text { when } \# \pi(i)=2 \\
\left(\frac{4}{9}\right)^{-} \text {when } \# \pi(i)=3 \\
\frac{16}{50} \text { when } \# \pi(i)=4
\end{array} \quad \text { while for each } i \in\{3,4\}\right. \text { we have } \\
& v_{i}(\pi)=\left\{\begin{array}{c}
\frac{1}{4} \text { when } \# \pi(i)=1 \\
\frac{4}{9} \text { when } \# \pi(i)=2 \\
\frac{9}{16} \text { when } \# \pi(i)=3 \\
\frac{16}{50} \text { when } \# \pi(i)=4
\end{array}\right. \text { according to which the set of efficient juris- }
\end{aligned}
$$

diction structures is $\{\{N \backslash\{i\},\{i\}\}: i \in N\} \cup\{\{K, N \backslash K\}: \# K=2\}$ while the grand jurisdiction is the only stable jurisdiction structure.

Remark 3.3 In the example that proves Theorem 3.3, switching to AE-FX renders the jurisdiction structures $\{\{N \backslash\{i\},\{i\}\}: i \in N\}$ individually stable while switching to FE- $A X$ renders the jurisdiction structures $\{\{K, N \backslash K\}: \# K=2\}$ individually stable. Under both switches, there remain stable partitions which are not efficient and efficient partitions which are not stable. So the welfare theorems fail to hold over $E$ under AE-FX and FE-AX as well.

The next theorem shows that tightening the membership property right code further, recovers one of the welfare theorems. ${ }^{17}$

Theorem 3.4 Given any $e \in E$, under $A E$ - $A X$, efficient jurisdiction structures are individually stable. However, there exists $e \in E$ where, under $A E-A X$, individually stable jurisdiction structures are not efficient.

Proof. Take any $e \in E$ and let AE-AX be the membership property right code. Take any $\pi \in \Pi$ which is not individually stable. So there exists $i \in N$ and $K \in(\pi \backslash\{\pi(i)\}) \cup\{\varnothing\}$ such that we have $v_{i}\left(F_{i, \pi(i), K}(\pi)\right)>v_{i}(\pi)$ and $v_{j}\left(F_{i, \pi(i), K}(\pi)\right) \geq v_{j}(\pi) \forall j \in \pi(i) \cup K$. Moreover, $v_{j}\left(F_{i, \pi(i), K}(\pi)\right)=v_{j}(\pi) \forall j \in$ $N \backslash(\pi(i) \cup K)$. Hence, $\pi$ is not efficient, as it is Pareto dominated by $F_{i, \pi(i), K}(\pi)$. The existence of economies where, under AE-AX, stable jurisdiction structures are not efficient, follows from the conjunction of Remark 2.1 with Theorem 3.3 which establishes the existence of such economies under FE-FX.

It is worth noting that the examples we use to show our results exhibit a particular "convexity" structure. We say that the preference $u_{i}\left(x_{i}, y, k\right)$ of $i \in N$

\footnotetext{
${ }^{17}$ The first part of Theorem 3.4 below follows from Proposition 3 of Bogomolnaia and Jackson (2002) which shows, in a general hedonic setting, that all efficient coalition structures are "contractually individually stable". (See Footnote 12.)
} 
is crowding-convex iff given any $\left(x_{i}, y\right) \in \Re_{+} \times \Re_{+}$and any $k_{1}, k_{2} \in\{1, \ldots, n-$ $1\}$ with $k_{1}>k_{2}$, we have $u_{i}\left(x_{i}, y, k_{1}+1\right)-u_{i}\left(x_{i}, y, k_{1}\right) \geq u_{i}\left(x_{i}, y, k_{2}+1\right)-$ $u_{i}\left(x_{i}, y, k_{2}\right)$. We write $E^{\text {convex }} \subset E$ for the set of economies where individual preferences are crowding-convex.

In fact, the proofs of Theorems 3.2, 3.3 and 3.4 use economies with crowdingconvex preferences - hence all four theorems can be stated by replacing $E$ with $E^{\text {convex }}{ }^{18}$ However, the failure of the welfare theorems is not due to crowding-convexity. To see this, we define the corresponding "concavity" condition and say that the preference $u_{i}\left(x_{i}, y, k\right)$ of $i \in N$ is crowding-concave iff given any $\left(x_{i}, y\right) \in \Re_{+} \times \Re_{+}$and any $k_{1}, k_{2} \in\{1, \ldots, n-1\}$ with $k_{1}>k_{2}$, we have $u_{i}\left(x_{i}, y, k_{1}+1\right)-u_{i}\left(x_{i}, y, k_{1}\right) \geq u_{i}\left(x_{i}, y, k_{2}+1\right)-u_{i}\left(x_{i}, y, k_{2}\right)$. We write $E^{\text {concave }} \subset E$ for the set of economies where individual preferences are crowding-concave.

Theorem 3.5 There exists $e \in E^{\text {concave }}$ where under FE-FX -hence also under tighter membership property right codes- stable jurisdiction structures are not efficient.

Proof. Consider $e \in E$ where $N=\{1,2,3\}$ and $\omega_{i}=1 \forall i \in N$. Letting $c_{1}=\left(\frac{16}{9}\right)^{+}$and $c_{2}=\left(\frac{9}{4}\right)^{-}$, preferences of agents are

$$
u_{i}\left(x_{i}, y, k\right)=\left\{\begin{array}{c}
x_{i} \cdot y \text { when } k=1 \\
\frac{x_{i} \cdot y}{c_{1}} \text { when } k=2 \\
\frac{x_{i} \cdot y}{c_{2}} \text { when } k=3
\end{array} \text { for each } i \in N \text {. So } e \in E^{\text {concave. }}\right. \text {. One }
$$

can check that this implies for each $\pi \in \Pi$ and each $i \in N$,

$$
v_{i}(\pi)=\left\{\begin{array}{c}
\frac{1}{4} \text { when } \# \pi(i)=1 \\
\left(\frac{1}{4}\right)^{-} \text {when } \# \pi(i)=2 \quad \text { according to which the partition } \\
\left(\frac{1}{4}\right)^{+} \text {when } \# \pi(i)=3
\end{array}\right.
$$

$\{\{i\}: i \in N\}$ is stable but not efficient under FE-FX - hence under tighter membership property right codes as well.

Theorem 3.6 There exists $e \in E^{\text {concave }}$ where under FE-FX, AE-FX or FEAX efficient jurisdiction structures are not stable.

Proof. Consider $e \in E$ where $N=\{1,2,3\}$ and $\omega_{i}=1 \forall i \in N$. Letting $c_{1}=\left(\frac{16}{9}\right)^{+}$and $c_{2}=\left(\frac{9}{4}\right)^{+}$, preferences of agents are

$$
u_{i}\left(x_{i}, y, k\right)=\left\{\begin{array}{c}
x_{i} \cdot y \text { when } k=1 \\
\frac{x_{i} \cdot y}{c_{1}} \text { when } k=2 \\
\frac{x_{i} \cdot y}{c_{2}} \text { when } k=3
\end{array} \text { for each } i \in\{1,2\} \text { and } u_{3}\left(x_{3}, y, k\right)=\right.
$$

$x_{3} . y$ for all $k \in\{1,2,3\}$. So $e \in E^{\text {concave }}$. One can check that this implies for each $\pi \in \Pi$ and each $i \in\{1,2\}, v_{i}(\pi)=\left\{\begin{array}{c}\frac{1}{4} \text { when } \# \pi(i)=1 \\ \left(\frac{1}{4}\right)^{-} \text {when } \# \pi(i) \geq 2\end{array}\right.$ while

\footnotetext{
${ }^{18} \mathrm{As}$ a matter of fact, the economies in the proof of Theorem 3.2 do not fit the definition of crowding-convexity. However, they can easily be rendered crowding-convex (by making $M$ increase with the number of agents within a coalition) without harming the results they establish.
} 
$v_{3}(\pi)=\left\{\begin{array}{c}\frac{1}{4} \text { when } \# \pi(3)=1 \\ \frac{4}{9} \text { when } \# \pi(3)=2 \\ \frac{9}{16} \text { when } \# \pi(3)=3\end{array}\right.$ according to which all jurisdiction structures but $\{\{1,2\},\{3\}\}$ are efficient. On the other hand, under FE-FX all jurisdiction structures; under AE-FX all jurisdiction structures but $\{\{1\},\{2\},\{3\}\}$; under FE-AX $\{\{1\},\{2\},\{3\}\}$ and $\{\{1,2\},\{3\}\}$ fail stability.

\section{Results: Coalitional Stability}

We first remark the efficiency of coalitionally stable jurisdiction structures independent of the membership property right code.

Remark 4.1 Under any $C=\left\{C_{i, K, L}\right\}$, a jurisdiction structure $\pi \in \Pi$ which is not efficient, fails coalitional stability as $N$ blocks $\pi$ by a $\rho$-move where $\rho$ Pareto dominates $\pi$ while $(\pi(i) \backslash N) \cap C_{i, \pi(i), \emptyset}=\emptyset$ for all $i \in N$. However the existence of coalitionally stable jurisdiction structures depends on $C$. For example, we know through the example given in Section 2.3 that, under FE-FX, individually stable (hence coalitionally stable) jurisdiction structures may fail to exist. Nevertheless, as we show below, when the membership property right code is tightened to AE-AX, every efficient jurisdiction structure is coalitionally stable. This ensures the existence of coalitionally stable jurisdiction structures, as efficient jurisdiction structures always exist. ${ }^{19}$

Theorem 4.1 Let $C=\left\{C_{i, K, L}\right\}$ be determined by $A E-A X$. Take any $e \in E$. Every efficient jurisdiction structure $\pi \in \Pi$ is coalitionally stable.

Proof. Let $C=\left\{C_{i, K, L}\right\}$ be determined by AE-AX. Take any $e \in E$ and any $\pi \in \Pi$ which is not coalitionally stable. If $\pi$ is not individually stable, then $\pi$ fails efficiency by Theorem 3.4. Now let $\pi$ be individually stable. So there exist $S \in 2^{N} \backslash\{\emptyset\}$ and $\rho \in \Pi(S)$ such that $v_{i}\left(F_{S, \rho}(\pi)\right) \geq v_{i}(\pi) \forall i \in S$ while $v_{i}\left(F_{S, \rho}(\pi)\right)>v_{i}(\pi)$ for some $i \in S$. Moreover, given any $i \in S$, we have $v_{j}\left(F_{S, \rho}(\pi)\right) \geq v_{j}(\pi) \forall j \in(\pi(i) \backslash S) \cap C_{i, \pi(i), \emptyset}$. Finally, $v_{j}\left(F_{S, \rho}(\pi)\right)=v_{j}(\pi)$ $\forall j \in N \backslash \bigcup_{i \in S} \pi(i)$. Hence, $\pi$ is not efficient, as it is Pareto dominated by $F_{S, \rho}(\pi)$.

Remark 4.2 Theorem 4.1 fails to hold under $A E-F X$ or FE-AX, as we know, from Remark 3.2, that these membership property right codes admit efficient jurisdiction structures which fail individual stability, hence coalitional stability.

The conjunction of Remark 4.1 and Theorem 4.1 leads to the following corollary:

Corollary 4.1 Let $C=\left\{C_{i, K, L}\right\}$ be determined by $A E-A X$. Take any $e \in E$. A jurisdiction structure $\pi \in \Pi$ is coalitionally stable if and only if $\pi$ is efficient.

${ }^{19}$ see Footnote 15. 


\section{Closing Remarks}

We consider the strategic formation of jurisdictions where an impure public good is locally produced. We show the existence of economies where individually stable jurisdiction structures and efficient jurisdiction structures form (non-empty) disjoint sets. This negative result is under the membership property right code which allows free entry to and free exit from jurisdictions. However, changing the code to approved entry-approved exit ensures the coalitional (hence individual) stability of efficient jurisdiction structures. As efficient jurisdiction structures always exist, we thus guarantee the existence of coalitionally stable jurisdiction structures. Moreover, every coalitionally stable jurisdiction structure is efficient. As a result, under approved entry-approved exit, the set of coalitionally stable jurisdiction structures and efficient jurisdiction structures coincide - hence rehabilitating the Tiebout (1956) conjecture. Some of these results follow, either intuitively or formally, from the vast literature ${ }^{20}$ on hedonic coalition formation and/or local public good production. However, as to our knowledge, Theorem 4.1 and Corollary 4.1 -which indeed hold for any general hedonic setting à la Bogomolnaia and Jackson (2002)- have not been previously established.

We owe our positive results to a design of rights à la Sertel $(1992,1998)$. Actually, there are previous explorations where certain concepts related to membership property rights have been incorporated into the definition of stability. ${ }^{21}$ However, we followed Sertel (2003) who advises to separate the membership property right code from the definition of stability so as to use the design of rights as a tool to improve social outcomes. This has been a useful advice regarding the production of local public goods and we believe, it can pave the way to further positive results in various other coalition formation contexts.

\section{References}

Asan, G. and Sanver, M. R., (2003) Coalition structural games and the voluntary provision of public goods, in Advances in Economic Design (eds. M.R. Sertel and S. Koray), Springer-Verlag, Berlin, 251-259.

Banerjee, S., Konishi, H., and Sonmez, T. (2001) Core in a simple coalition formation game, Social Choice Welfare 18, 135-153.

Bergstrom, T., Blume, L. and Varian, H. (1986) On the private provision of public goods. Journal of Public Economics 29: 25-49

Bewley, T. (1981), A critique of Tiebout's theory of local public expenditure. Econometrica, 49: 713-740.

Bogomolnaia, A., Jackson, M. O. (2002) The stability of hedonic coalition structures. Games and Economic Behavior 38 (2): 201-230

Buchanan, J. M. (1965) An economic theory of clubs. Economica 32: 1-14

\footnotetext{
${ }^{20}$ which we non-exhaustively cite

${ }^{21}$ such as in Bogomolnaia and Jackson (2002). See Footnote 12.
} 
Buchanan, J. M., Goetz C. (1972), Efficiency limits of fiscal mobility, Journal of Public Economics, 1: 25-45

Conley, J.P. and Konishi, H. (2002), Migration proof Tiebout equilibrium: Existence and asymptotic efficiency, Journal of Public Economics, 86, 243-262.

Drèze, J., Greenberg J. (1980) Hedonic coalitions: Optimality and stability. Econometrica 48: 987-1003

Eren, N. İ. (1993), Coalition structural games and stability under membership property right axioms. Unpublished M.A. Thesis, Boğaziçi University

Flatters, F., Henderson V., Mieszkowski P. (1974), Public goods, efficiency and regional fiscal equalization. Journal of Public Economics, 3: 99-112

Greenberg, J., (1983) Local public goods with mobility: Existence and optimality of a general equilibrium under. Journal of Economic Theory 30: 17-33

Greenberg, J., Weber, S. (1986) Strong Tiebout equilibrium under restricted preference domain. Journal of Economic Theory 28: 101-117

Greenberg, J., Weber, S. (1993) Stable coalition structures with unidimensional set of alternatives. Journal of Economic Theory 60: 62-82

Guesnerie, R., Oddou, C. (1981) Second best taxation as a game. Journal of Economic Theory 25: 67-91

Konishi, H. (1996) Voting with ballots and feet: Existence of equilibrium in a local public good economy. Journal of Economic Theory 68: 480-509.

Konishi, H., le Breton, M. and Weber, S. (1998) Equilibria in finite local public good economies. Journal of Economic Theory 79: 224-244.

McGuire, M. (1974) Group segregation and optimal jurisdictions. Journal of Political Economy 82: 112-132

Nizamogullari, D. and I. Ozkal-Sanver (2007), Coalitional stability and efficiency of partitions in matching problems, unpublished manuscript.

Ozkal-Sanver, I (2005) Stability and efficiency of partitions in matching problems. Theory and Decision 59 (3) : 193-205

Richter, D. K. (1982), Weakly democratic regular tax equilibria in a local public goods economy with perfect consumer mobility, Journal of Economic Theory 27: 137-162

Sertel, M. R. (1982) Workers and Incentives (Contributions to Economic Analysis, No.140). North-Holland, Amsterdam, New York

Sertel, M. R. (1992) Membership property rights, efficiency and stability. Boğaziçi University Research Papers

Sertel, M. R. (1998) Designing rights: Invisible hand and decentralizability theorems. ASSET Lecture delivered at the annual meeting of ASSET in Bologna

Sertel, M. R. (2003), Designing Rights, mimeo

Sertel, M.R. and M. Yildiz (1998), The Lindahl solution with changing population and resources, Mathematical Social Sciences, 35 (2),151-163.

Tiebout, C. (1956) A pure theory of local expenditures. Journal of Political Economy 64: 416-424

Wooders, M. H. (1978) Equilibria, the core and jurisdictions structures in economies with a local public good. Journal of Economic Theory 18: 328-348 